http://dx.doi.org/10.21611/qirt.1994.017

\title{
Infrared polarization thermometry \\ using an imaging radiometer
}

by BALFOUR L. S. *

"EORD, Technion Research \& Development Foundation Ltd, Haifa 32000, Israel.

\begin{abstract}
This paper describes a method to determine absolute temperatures of surfaces based on the thermal infrared polarization of the radiant beam emanating from the surface. No knowledge of the surface emissivity is required but a known source of radiation is required to be reflected off the surface. Results of measurements from two samples are presented.
\end{abstract}

\begin{tabular}{|c|c|c|c|c|c|}
\hline \multicolumn{2}{|c|}{ Nomenclature } & \multicolumn{2}{|r|}{ Greek symbols } & \multicolumn{2}{|c|}{ Subscripts } \\
\hline$L$ & Radiance (W/cm²/str) & $\varepsilon$ & emissivity & $p$ & parallel component \\
\hline $\mathrm{T}$ & Temperature(K) & $\lambda$ & wavelength(microns) & $\mathbf{s}$ & normal component \\
\hline $\mathrm{P}$ & Planck function & & & a & ambient \\
\hline
\end{tabular}

\section{Introduction}

The thermal infrared radiation emanating from a surface is a function of several parameters such as its temperature, emissivity, roughness and direction of emission with respect to the surface normal. Surfaces that are not ideally black have an emissivity value less than unity. Consequently the beam of radiation emanating from the surface is comprised of two parts namely a self emitted part and a reflected part.The surface temperature information is contained in the self emitted part only and it is not possible to separate the emitted part only from the total beam. Usually in remote measurements one has to assign an emissivity value to the surface and specify the effective temperature of the surroundings reflected by the surface in order to deduce the surface temperature. Moreover the surface emissivity usually has a spectral structure and consequently affects the acuracy of value of the deduced temperature

In this paper we present a novel method to determine the absolute temperature of a surface without requiring any knowledge of the surface emissivity. The method is based on measuring the linear polarization of the radiation beam which is a function of the difference in temperature between the surface and the reflected surroundings. As will be shown the method is applicable to any surface which is not ideally diffuse or Lambertian in nature. 


\section{Principle of the method}

The polarization in a beam of infrared radiation emanating from an opaque dielectric surface is due to the change in the complex refractive index at the surface-air interface.The distribution of the radiant power with respect to the orthogonal components parallel and perpendicular to the plane of vision is governed by the Fresnel relations [1]. The plane of vision is defined as the plane containing the line of sight to the surface and the surface normal. The Fresnel relations describe the polarizations of the specularly reflected and transmitted (emitted) components and their angular dependence is shown in figure 1.

In the thermal infrared region the radiation from a surface contains a self emitted part and a reflected part. One can further consider this radiation to be a mixture of unpolarized and polarized radiation giving rise to a partially polarized beam whose degree of polarization depends upon the specularity of the surface. The temperature difference between the surface and the reflected surroundings can be related to the net linear polarization of the emanating beam as follows.

Considering the specular part, the polarized radiance emanating from the surface in the parallel and perpendicular planes is given by

$$
\begin{aligned}
& L_{p}^{\lambda}=\varepsilon_{p}^{\lambda} \cdot P(\lambda, T)+\left(1-\varepsilon_{p}^{\lambda}\right) \cdot P\left(\lambda, T_{a}\right) \\
& L_{s}^{\lambda}=\varepsilon_{s}^{\lambda} \cdot P(\lambda, T)+\left(1-\varepsilon_{s}^{\lambda}\right) \cdot P\left(\lambda, T_{a}\right)
\end{aligned}
$$

In these relations the first term on the right hand side represents the self emitted part and the second the reflected part. By passing the radiation through an ideal linear polarizer one can selectively transmit either the parallel or perpendicular component. The part of the unpolarized radiation transmitted by the linear polarizer is the same for both orientations. Hence the difference of the transmitted polarized radiances is given by

$$
L_{p}^{\lambda}-L_{s}^{\lambda}=\left(\varepsilon_{p}^{\lambda}-\varepsilon_{s}^{\lambda}\right) \cdot\left(P(\lambda, T)-P\left(\lambda, T_{a}\right)\right)
$$

For all view directions away from the normal $\left(\varepsilon_{\mathrm{p}}^{\lambda}-\varepsilon_{\mathrm{s}}^{\lambda}\right)>0$

Hence $\quad L_{p}^{\lambda}-L_{s}^{\lambda}=0 \quad$ only when $T=T_{a}$.

This is the basis on which polarization thermometry is carried out [2]. By reflecting a blackbody source off the surface and measuring the difference in the polarized radiances of the beam containing both emitted and reflected components we obtain a null value when the source temperature is identical to the surface temperature.

\section{Measurements}

The basic instrument used to measure polarized radiances was the AGA 780 LW imaging radiometer. This instrument was modified to measure polarized radiances by inserting a linear polarizer in the optical path to the detector. The response of most optical instruments is sensitive to a greater or lesser degree to the orientation of the linearly polarized beam and hence a radiometric calibration is necessary. This calibration was performed for the two orthogonal orientations of the polarizer and a typical calibration curve is shown in figure 2. A linear regression yields slope and offset values which are used to convert the instrument output to radiance values. 
Polarization thermometry measurements were carried out on a painted copper block using two different experimental setups. In the first case the copper block was mounted on a hot plate source and its temperature was stabilized using a temperature controller. A blackbody source was reflected off the painted surface and the thermogram of the surface recorded by a computerized image capture system. The surface was maintained at a temperature of $60 \mathrm{C}$ as measured by an embedded resistance thermometer and the blackbody source temperature was varied in stages from $40 \mathrm{C}$ to $80 \mathrm{C}$. At each stage, for a given blackbody temperature the polarized radiance images for the two orthogonal positions were recorded. In this setup the ambient surroundings were not homogeneous with regard to temperature.

In the second setup the copper block was mounted in a box and viewed through an aperture in the wall of the box. In this case the temperature of the copper block was varied using a thermoelectric element and its temperature maintained by a controller.The block was painted with a different colour(in the visible) and its temperature measured using an embedded resistance thermometer. The temperature of the box enclosure was measured using a thermocouple probe. As in the previous case polarized image pairs were recorded.

\section{Results}

The difference between the parallel and perpendicularly polarized radiances normalized to their sum (i.e the degree of polarization) is plotted against the difference in temperatures between the surface and the surroundings. The results of the first setup are shown in figure 3. We see that for surface temperatures higher than the blackbody source the difference is positive and when lower than the blackbody source it is negative. The radiance null difference point corresponds to the temperature null difference point within the limits of the experimental error. Near the null point we note an increase in the radiance difference when the surface temperature is below the blackbody temperature. This behaviour is possibly due to a fluctuation in the surface temperature of the blackbody source at the time of measurement.

The results of the experiment with the homogenous surroundings is shown in figure 4 . In this case also we see that the null in radiance difference corresponds to a null in temperature difference. Moreover no abnormal behaviour is noted both below and above the null point.

\section{Conclusions}

The proposed method gives results in agreement with the simple Fresnel model predicting a null difference in polarized radiation when the surface and reflected sources are at the same temperature. An attractive feature of the method is the ability to measure true surface temperatures without any knowledge of the surface emissivity.

\section{REFERENCES}

[1] BENNET (J.M.), BENNET (H.E.) "Polarization" - Handbook of Optics, Driscoll, W. G. and Vaughan, W. eds, McGraw-Hill Book, 1978, section 10

[2] RUDOLPH (R.G.) - "Polaradiometer: A Polarization technique for Temperature measurement" - Theory and Practice of Radiation Thermometry, Diwitt, D.P. and Nutter, G.D.eds, John Wiley \& Sons, 1988, chapter 9. 
http://dx.doi.org/10.21611/qirt.1994.017

\section{FRESNEL COEFFICIENTS REFLECTED AND EMITTED COMPONENTS}

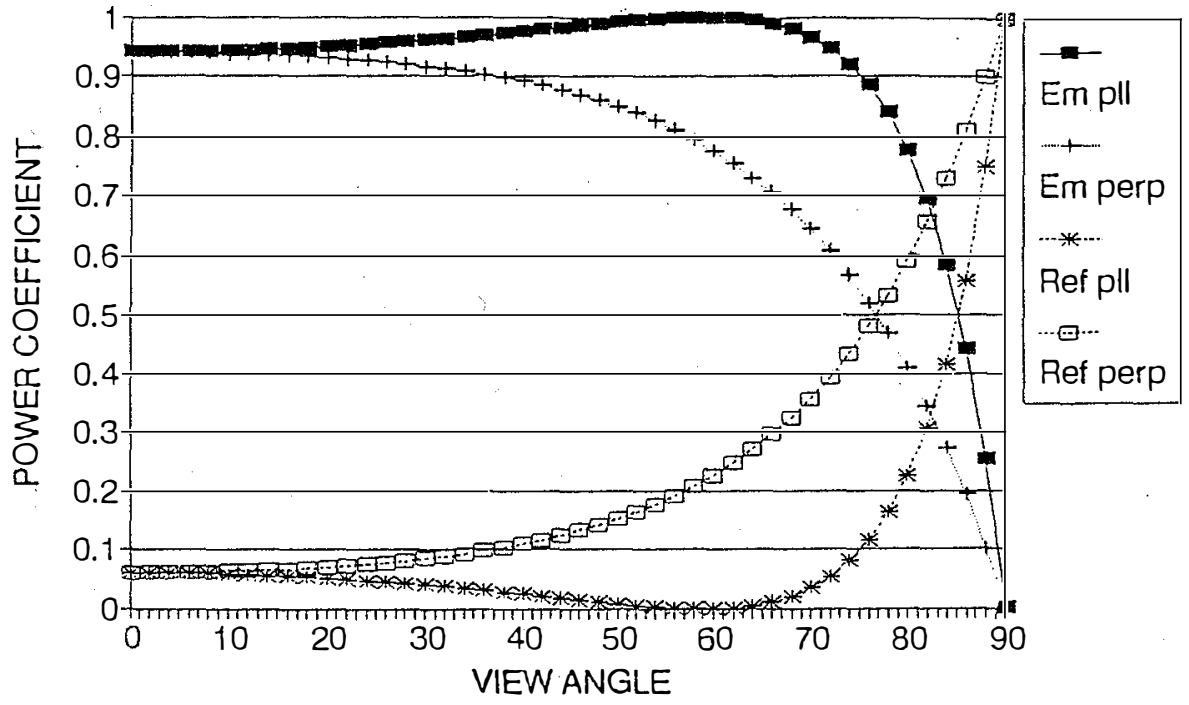

Fig 1. The angular dependence of the Fresnel coefficients for reflected and transmitted radiation from an opaque dielectric surface.

\section{Polarimeter calibration curve} transmission direction: perpendicular

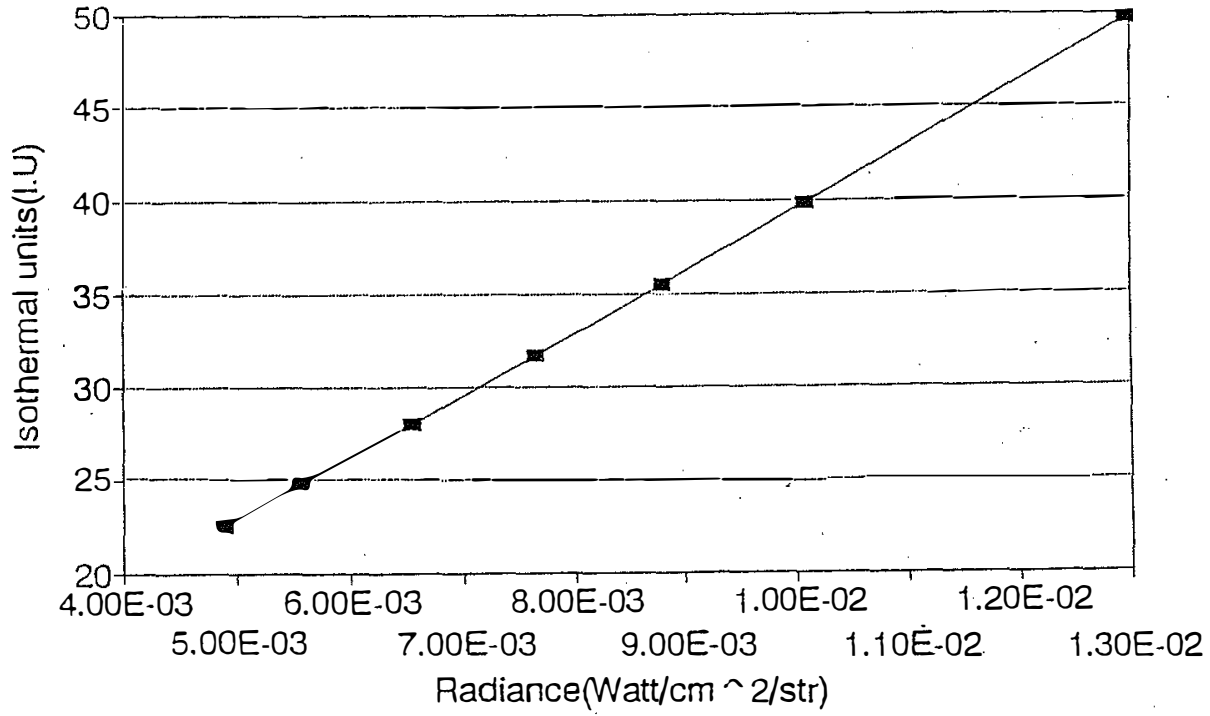

Fig.2. The radiometric calibration curve of the AGA imager with a linear polarizer. 
http://dx.doi.org/10.21611/qirt.1994.017

\section{Degree of Polarization vs delta Temp BB source reflected off surface at $61 \mathrm{C}$}

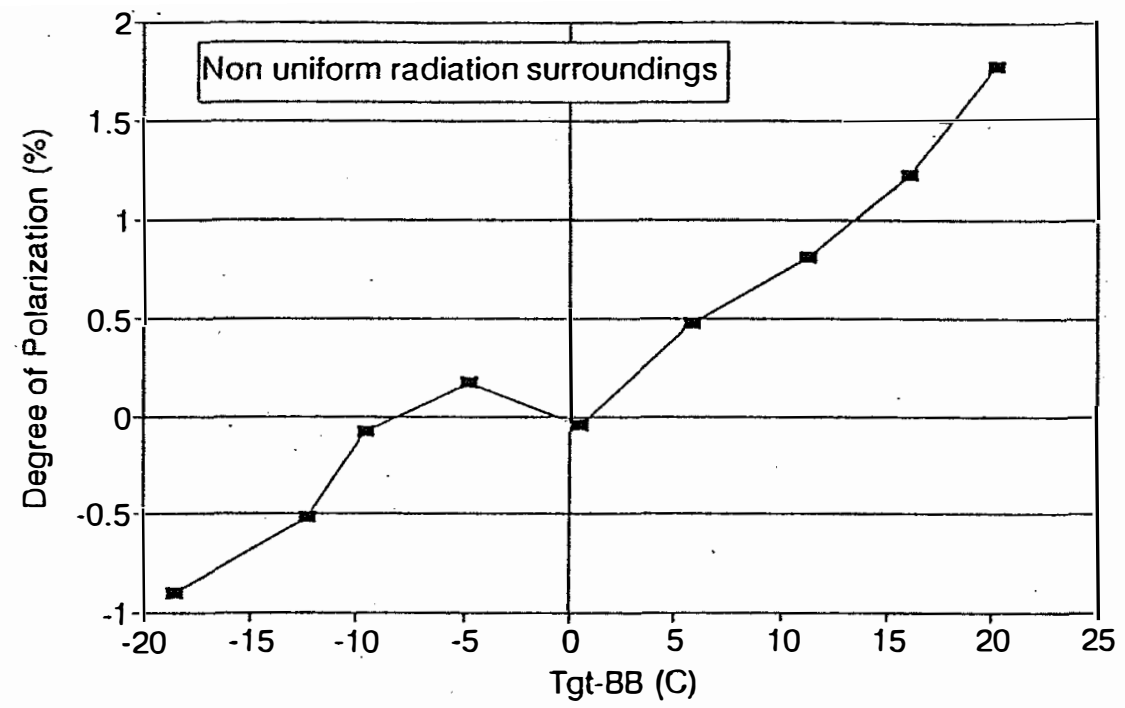

Fig.3. The degree of linear polarisation versus temperature difference between the painted sample surface and the reflected blackbody source.

\section{Degree of Polarization vs delta Temp}

Uniform radiation surrounding at $\mathrm{T}=14 \mathrm{C}$

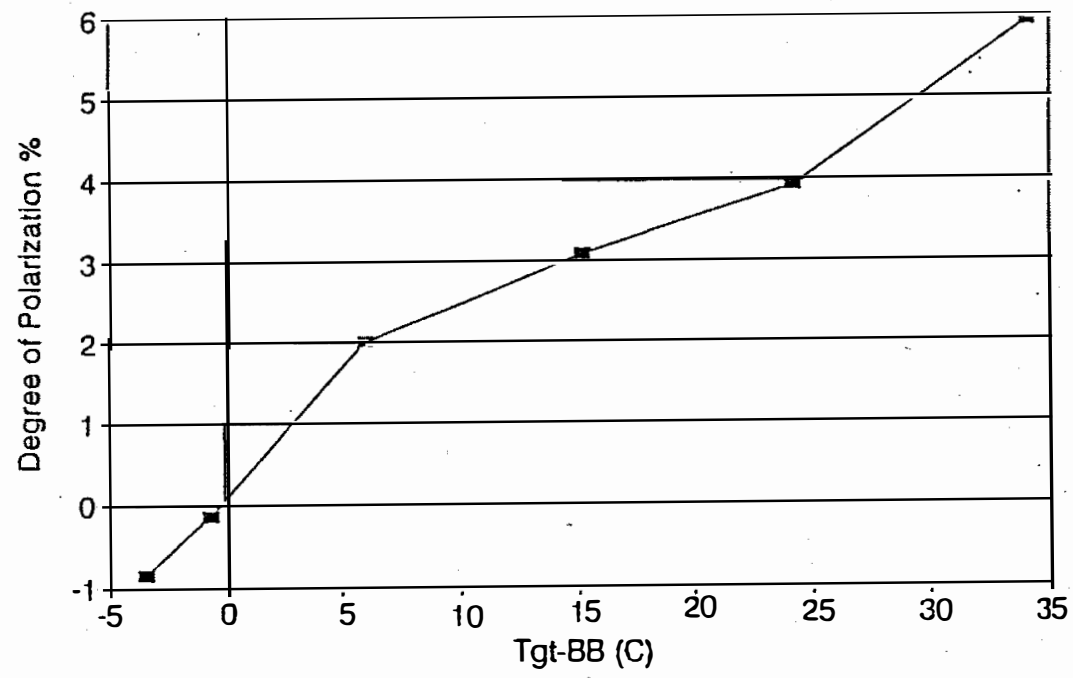

Fig.4. The variation of the degree of linear polarisation with temperature difference between the painted sample surface and the uniform box wall surroundings. 